\title{
Characterization and gene expression analysis of the cir multi-gene family of plasmodium chabaudi chabaudi (AS)
}

Jennifer Lawton', Thibaut Brugat ${ }^{1}$, Yam Xue Yan², Adam James Reid³, Ulrike Böhme ${ }^{3}$, Thomas Dan Otto ${ }^{3}$, Arnab Pain ${ }^{3,4}$, Andrew Jackson ${ }^{3}$, Matthew Berriman ${ }^{3}$, Deirdre Cunningham ${ }^{1}$, Peter Preiser ${ }^{2}$ and Jean Langhorne ${ }^{1^{*}}$

\begin{abstract}
Background: The pir genes comprise the largest multi-gene family in Plasmodium, with members found in $P$. vivax, $P$. knowlesi and the rodent malaria species. Despite comprising up to 5\% of the genome, little is known about the functions of the proteins encoded by pir genes. P. chabaudi causes chronic infection in mice, which may be due to antigenic variation. In this model, pir genes are called cirs and may be involved in this mechanism, allowing evasion of host immune responses. In order to fully understand the role(s) of CIR proteins during P. chabaudi infection, a detailed characterization of the cir gene family was required.
\end{abstract}

Results: The cir repertoire was annotated and a detailed bioinformatic characterization of the encoded CIR proteins was performed. Two major sub-families were identified, which have been named A and B. Members of each subfamily displayed different amino acid motifs, and were thus predicted to have undergone functional divergence. In addition, the expression of the entire cir repertoire was analyzed via RNA sequencing and microarray. Up to 40\% of the cir gene repertoire was expressed in the parasite population during infection, and dominant cir transcripts could be identified. In addition, some differences were observed in the pattern of expression between the cir subgroups at the peak of $P$. chabaudi infection. Finally, specific cir genes were expressed at different time points during asexual blood stages.

Conclusions: In conclusion, the large number of cir genes and their expression throughout the intraerythrocytic cycle of development indicates that CIR proteins are likely to be important for parasite survival. In particular, the detection of dominant cir transcripts at the peak of $P$. chabaudi infection supports the idea that CIR proteins are expressed, and could perform important functions in the biology of this parasite. Further application of the methodologies described here may allow the elucidation of CIR sub-family A and B protein functions, including their contribution to antigenic variation and immune evasion.

\section{Background}

An important feature of the malaria parasite is the export of proteins to the surface of infected red blood cells (iRBCs). Surface proteins or variant surface antigens (VSA) have been identified so far in three species infecting humans: Plasmodium falciparum, $P$. vivax and $P$. knowlesi, as well as in the rodent malaria parasites $P$. chabaudi and P. yoelii [1-5]. These proteins are implicated in antigenic variation and immune evasion, as well

\footnotetext{
* Correspondence: jlangho@nimr.mrc.ac.uk

'Division of Parasitology, MRC National Institute for Medical Research, London, UK Full list of author information is available at the end of the article
}

as parasite accumulation or sequestration in host tissues; features which may be critical in determining the outcome of malaria reviewed by $[6,7]$. Since VSAs are recognized by antibodies, they are also likely to be important targets for a protective immune response eg. $[8,9]$.

In most Plasmodium species VSAs are encoded by multi-gene families, usually located in sub-telomeric chromosome regions [2,10-12]. In P. falciparum, several VSA gene families have been identified such as var, rif, stevor and surf reviewed by [7]. Among them, the best characterized is the var gene family encoding PfEMP1 proteins, which is implicated in both antigenic variation
() Biomed Central 
and sequestration $[1,13]$. This family consists of 60 genes that can be grouped into several families (A-E) according to chromosomal location, coding and nonintergenic sequences, direction of transcription, and domain arrangements $[14,15]$. Similarly, the rif genes, the largest multi-copy gene family in P. falciparum (circa 150-200 genes), has been divided into subgroups $A$ and $B$ on the basis of sequence similarity $[16,17]$. Aand B-type RIFINs have different sub-cellular localizations, in that only the A-type RIFINs appear to be exported towards the surface of iRBCs [16]. Thus, Atype RIFINs may be more likely to play a role in the host/parasite relationship during the blood stages of $P$. falciparum.

Plasmodium interspersed repeat (pir) genes have been identified in Plasmodium vivax (vir), P. knowlesi (kir), P. berghei (bir), P. chabaudi (cir), and P. yoelii (yir) $[2,18,19]$. Together, pirs form the largest multi-gene family identified to date in Plasmodium species, and occupy up to $5 \%$ of the parasite genome. On completion of the P. vivax Salvador I genome sequence, a total of 12 vir sub-families were identified [20-22]. Similarly, 5 sub-families have been identified within the yir repertoire [23]. However, so far no function has been ascribed to the subgroups of yir and vir families.

Microarray studies carried out on $P$. vivax and $P$. yoelii suggest that approximately $50 \%$ of the $y i r$ and vir repertoires are expressed in a population of iRBCs during infection, with no evidence of preferential transcription according to their chromosomal location or phylogenetic sub-groups $[2,4,20,24]$. Surprisingly, considering the large proportion of yir genes transcribed in the parasite population, only 1-3 yirs are transcribed in a single iRBC [4]. This suggests that transcription of these genes is under tight control. The function(s) of PIR proteins, however, remain unknown.

Rodent malarias offer the only feasible system in which the contribution of PIR proteins to immune evasion and to sequestration and pathology can be examined. Of these, $P$. chabaudi is the only rodent species that naturally produces a chronic infection in mice. These chronic infections are likely to be perpetuated by antigenic variation [25-27]; however, the antigens involved have not yet been determined. P. chabaudi also exhibits other important features observed in human Plasmodium infections, including rosetting and adhesion to host endothelial cells $[5,28]$, and thus is an ideal model in which to investigate the role of PIR proteins.

The cir genes comprise the largest gene family in $P$. chabaudi, and are located in sub-telomeric regions of chromosomes [10,29]. Recently, restriction fragment length polymorphism (RFLP) analysis indicated that cir genes may have tissue specific patterns of expression
[30]. However, as such techniques are not gene specific, they are only able to provide clues about general cir expression. Furthermore, very little is known about the function of CIRs in stimulating or evading host immunity during $P$. chabaudi infection.

Here we describe two major sub-families within the cir repertoire of the recently completed P. chabaudi AS genome. Using an approach similar to that applied to the RIFIN repertoire [17], we found both conserved and sub-family specific amino acid motifs, and predict functional divergence between the proteins from different CIR sub-families.

We have analyzed in detail the expression of the entire cir repertoire via RNA sequencing (Illumina RNA-seq) and microarray. Up to $40 \%$ of the cir gene repertoire was expressed in the parasite population during infection, and dominant cir transcripts could be identified, with some differences in the pattern of expression between the cir subgroups. Finally, we found specific cir genes were expressed at different time points during asexual blood stages. Together these data will allow future investigation of the CIR family to elucidate their roles in the host/pathogen relationship.

\section{Methods}

\section{Ethics statement}

This study was carried out in strict accordance with the UK Animals (Scientific Procedures) Act 1986 and was approved by the Ethical Committee of the MRC National Institute for Medical Research, and the British Home Office (PPL: 80/2538).

\section{Annotation of cir genes}

cir genes were annotated using Artemis release 11 $[31,32]$ onto the eight-fold coverage assembly of the Plasmodium chabaudi chabaudi AS genome [33]. Putative coding sequences containing conserved features of previously published cir genes identified from the threefold coverage genome assembly [34]. These were then searched for similarity to the pir superfamily genes via a combination of basic local alignment search tool (BLAST) and Hidden Markov Model (HMM) on PIR super-family proteins [CIR_BIR_YIR (PF06022)], available in the PFAM database [35], following previously described methods $[35,36]$.

\section{Detection of conserved motifs}

Motif Elicitation analysis (MEME, [37]) was used to identify up to 20 conserved amino acid motifs within the CIR repertoire. The average motif locations were identified and plotted onto each gene. WebLogos were generated with the MEME program, using an adaptation of the WebLogo software [38]. 


\section{Analysis of sequence similarity}

Amino acid sequences of 183 cir genes were aligned using the MUltiple Sequence Comparison by LogExpectation algorithm (Muscle, [39]). Sequences aligning poorly with the other CIRs were excluded from the alignment and regions containing large insertions were deleted (Additional file 1). The sequence similarity along the alignment is attached in Additional file 2.

Since phylogenetic trees only represent a bifurcating lineage and imply phylogenetic relationships, reticulate networks were created within the program Splitstree 4.0 [40]. All networks used the algorithms NeighborNet [41] for calculation of distances and Equal angle [42] for calculation of splits. 500 bootstrap replicates were generated (Additional file 3).

To support the network, a phylogenetic tree was also constructed using the Maximum Likelihood method from the PhyML server [43]. Here, the evolutionary model applied was Le Gascuel (LG) [44], and the branch support was calculated by approximate likelihood ratio test (aLRT) [45]. Three YIR [46] and three BIR sequences (Ulrike Böhme, WTSI, personal communication) were added to the CIR alignment to allow a root to be placed within this tree (Additional file 4).

Clades identified by both methods with high branch support values contained highly similar CIR sequences. Small clades within each major sub-family were denoted numerically: A1-A5 and B1-B4. Members of each clade are tabulated in Additional file 5.

\section{Detection of phylogenetic incompatibilities between cir genes}

Phylogenetic incompatibilities within the alignment of 183 CIRs, and each identified sub-family, were analyzed using the pairwise homoplasy index (PHI) in Splitstree v4.0 [40].

Phylogenetic profiling was used to detect phylogenetic inconsistencies between four cir DNA sequences, selected at random, using a hidden Markov model method within the TOPALi platform v2.5 $[47,48]$. The probability of generating each of the three possible tree topologies for the four sequences was modelled in a given 100 nucleotide window. Possible recombination breakpoints were identified where the most probable topology altered at different positions along the alignment.

Five cir quartets, chosen at random, were analyzed per clade by each method described above (Additional file 6).

\section{Function shift analysis}

The alignment of 183 CIRs was split into two files containing only A- and B-type CIRs. The two alignments were then used to apply the 'FunShift' methodology [49], and predict whether these 2 groups of proteins may perform different functions. Positions containing only gaps in a subfamily were not counted.

The method used was the same as described previously by Abhiman and Sonnhammer [49] with a few modifications. Rate-Shifting Sites (RSS) were defined as positions conserved in one sub-family but variable in the other, and were identified using the likelihood ratio test (LRT) program [50]. The U-values generated by this program indicate the likelihood of rate change for each position in the alignment between the two sub-families. U-values above 4.0 were considered significant at the $5 \%$ significance level, as previously described [50].

Conservation-Shifting Sites (CSS) were defined as positions that were conserved in both groups, but containing different residues in each. CSS were detected using the method developed by Abhiman and Sonnhammer [49]. This calculates a Z-score based on the normalized cumulative relative entropy at each position of the alignment, between the two sub-families. Z-scores exceeding 0.5 per alignment position were considered significant [49].

CSS and RSS are plotted within a sub-section of the alignment in Additional file 7.

\section{Mice and parasites}

Female BALB/c and C57BL/6 mice aged 6-8 weeks were obtained from the specific pathogen-free unit at the MRC National Institute for Medical Research (NIMR), London. For experimental purposes, mice were housed conventionally with sterile bedding, food and irradiated water on a 12 hour light-dark cycle.

A cloned line of Plasmodium chabaudi chabaudi (AS) was used in this study [51]. Stabilates were cryo-preserved in blood from BALB/c mice. To obtain parasites for experimental infection, an aliquot of the stabilate was injected intraperitoneally (i.p.) into immunodeficient $\mathrm{BALB} / \mathrm{c} R A G 2^{-/-}$mice [52]. Blood was taken from the donor mice 7 days after infection and experimental mice were infected by injecting $10^{5}$ infected erythrocytes i.p. Parasitaemia was monitored by examination of Giemsastained blood films as previously described [53].

Blood was collected from each mouse by cardiac puncture under terminal anaesthesia into Krebs saline (114 mM NaCl, $4.57 \mathrm{mM} \mathrm{KCl}, 1.15 \mathrm{mM} \mathrm{MgSO} 4)$ containing $0.2 \%$ glucose and $25 \mathrm{U} / \mathrm{ml}$ heparin (Leo Pharmaceuticals) 7 days after infection. Leukocytes were removed via Plasmodipur filtration (Euro-Diagnostica) according to manufacturer's instructions. Blood was then stored at $-80^{\circ} \mathrm{C}$ in TRIZOL reagent (Invitrogen) for subsequent RNA extraction.

\section{RNA extraction}

RNA was extracted from P. chabaudi infected blood samples by guanidinium thiocyanate-phenol-chloroform 
extraction according to standard methods [54] and DNase digested using Turbo DNAse (Ambion) according to the manufacturer's instructions.

\section{Microarray hybridization and analysis}

P. chabaudi AS is a highly synchronous parasite for which development in the blood follows its host's circadian rhythm. Twelve time-points were then collected; one every two hours, to cover the entire 24 h cycle of blood stage development. At the peak of parasitaemia, one mouse was sacrificed at each time point and thin blood films were made and stained with Giemsa for optical microscopy. The pan-rodent microarray was designed using the OligoRankPick program as previously described [55]. The RNA preparation, Cy-dye coupling to cDNA, hybridization and slide scanning were performed as described by Bozdech and colleagues [56].

Data processing and analysis (including the Fast Fourier Transform) were carried out as described by Bozdech and colleagues [24]. The phaseogram (Figure 7) contains genes with $>1.7 \log _{2}$ ratio of change in mRNA abundance across the IDC. A list of identified genes can be found in Additional file 8.

\section{RNA sequencing}

$10 \mu \mathrm{g}$ of $P$. chabaudi total RNA obtained from BALB/c and $\mathrm{C} 57 \mathrm{BL} / 6$ mice was used for this analysis. RNA sequencing was performed using an Illumina GAIIx following the methodology described by Otto and colleagues [57] and was used to create 76 bp paired-end reads. TopHat [58] was used to map reads against the Plasmodium chabaudi chabaudi AS reference genome [33], with maximum intron size set to 10000 and inner-mate distance set to 100. Gene expression levels (RPKM) were calculated as defined by Mortazavi and colleagues [59] with minor modifications. Non-uniquely mapping reads were excluded and read-length windows of protein-coding regions that were non-unique were excluded from the gene length term used in calculating RPKM.

Each sequencing run contained different amounts of noise (eg. reads mapping to generally unexpressed parts of the genome: introns and intergenic regions). Therefore we calculated RPKMs over 500 bp windows of exonic and intronic sequences on chromosome 14. For each sequencing run, we took an RPKM cutoff above which only $10 \%$ of intron sequences were expressed. A list of identified genes can be found in Additional file 9, with the threshold calculation in Additional file 10. Legends for Additional files 1-10 can be found in Additional file 11.

A Kolmogorov-Smirnov test [60] was used to compare the distribution of cir sub-groups according to their level of expression.

\section{Results}

I) Bioinformatic analysis of the CIR multi-gene family

\section{a) Identification of cir genes}

Completion of the $P$. chabaudi AS genome sequencing and revised assembly allowed the initial cir annotation to be manually revisited. Conserved features from the initially identified cir genes [18,29], such as the relative exon lengths, splice sites and amino acid sequences were used to identify putative cir coding sequences in the assembled contigs. 117 cir genes were first identified and manually annotated. A hidden Markov model (HMM) was then constructed on the basis of the identified cir repertoire and was used to detect more divergent cir genes, bringing the total of identified cirs to 196 [33], including 3 additional cir-like genes, containing some but not all expected features of cir family members.

Only three partial cir genes were identified, arising from the few unresolved contig assemblies for the $P$. chabaudi AS genome. Three long cir genes were also identified, containing an extended first exon. The majority of cir genes contained one predicted TM domain including some of the divergent cirs.

\section{b) Sequence similarity of CIR proteins}

In order to investigate similarity between CIRs, the amino acid sequences identified during cir gene annotation were aligned using Muscle [39], and refined manually (Additional file 1a). Upon addition of more divergent cir genes to the repertoire, 16 sequences aligned poorly with the majority of CIRs and were excluded from the analysis (tabulated in Additional file 1b). These included the three partial CIRs, eight CIR sequences with low $\mathrm{C}$ - or $\mathrm{N}$-terminal similarity to other members of the repertoire, two CIRs encoded by genes with an atypical structure and three sequences which had been identified as cir-like genes, containing some but not all features of cir genes.

The sequence similarity of the alignment was determined using Plotcon [61]. The most conservation was found between amino acids 75-120 and 350-385 (Additional file 2).

To determine the relationships between CIR sequences, a network was created (Figure 1, Additional file 3), using the NeighborNet and Equal Angle algorithms $[41,42]$ in the Splitstree program [40]. This methodology prevented bias from inferred evolutionary relationships, common to phylogenetic analyses [62]. Assumptions of linear evolution are not truly appropriate in the analysis of multi-gene family members which are likely to undergo frequent recombination, as the var and sicavar genes are known to do $[19,63,64]$. Instead, production of a network enables visualization of boxlike structures (reticulations), where recombination may 


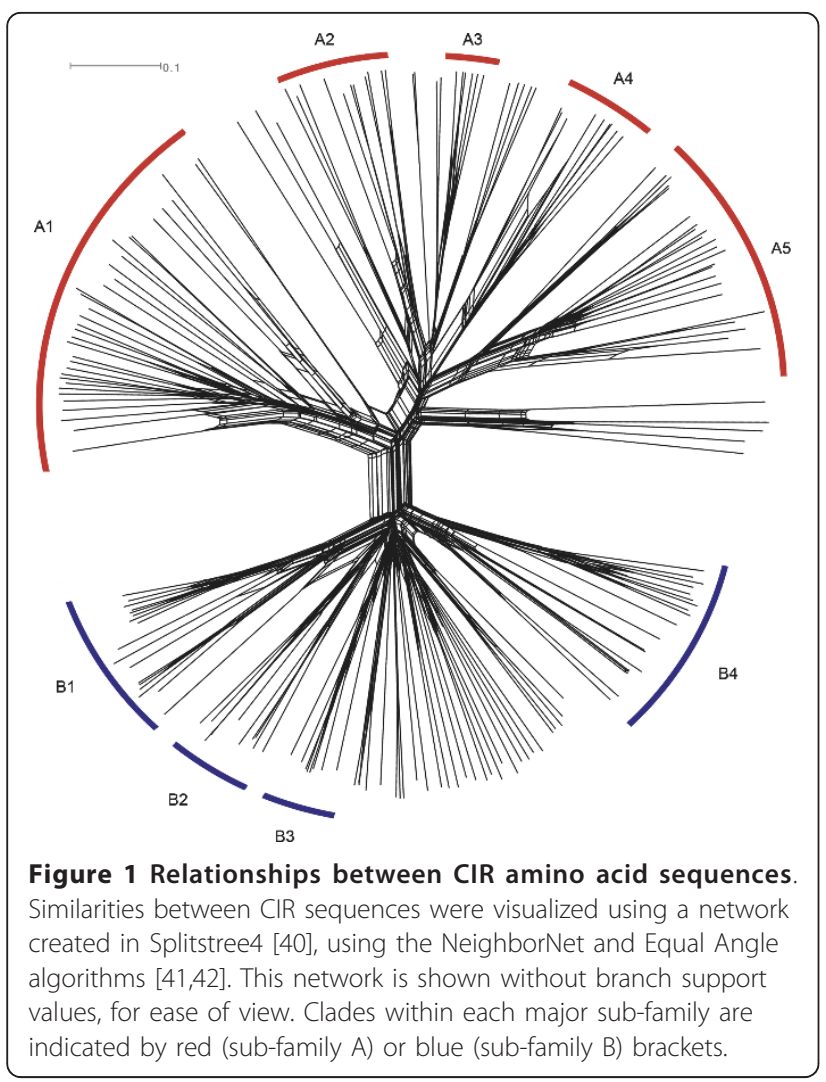

have occurred between cirs and linear evolution cannot be assumed (reviewed by [62]).

Figure 1 shows two distinct sub-families, each of which comprises multiple smaller clades, the members of which are shown in Additional file 5. The large subfamily A contained more divergent CIR sequences, many of which were identified by the CIR HMM. Five clades containing more than five CIR sequences were present within this sub-family: A1-A5 (highlighted in red, Figure 1), of which clade A1 was the most distinct. Sub-family B was comprised of the most conserved CIR sequences, and could also be further sub-divided into 4 clades: B1-B4 (highlighted in blue, Figure 1). The definition of the A1 group as a part of sub-family A was further reinforced by the creation of a phylogenetic tree to support the clades identified by the network in Figure 1. The Maximum Likelihood tree contained the same sub-families and smaller clades as observed in Figure 1, even after three YIR and three BIR sequences ([46], Böhme et al., unpublished) were added to the CIR alignment to enable a root to be placed (Additional file 4 TreeBase study accession URL http://purl.org/phylo/treebase/phylows/study/TB2: S12458 [65-67]). The clade A1 continued to cluster within CIR sub-family A, indicating that these CIR sequences were more similar to each other than to members of CIR sub-family B.
In addition, the visualization of CIR sequence relationships using network methodology indicated phylogenetic inconsistencies within the cir repertoire, as many boxlike structures (reticulations) were present (Figure 1). Across the whole cir repertoire, different regions of cir genes were found to display different phylogenetic relationships with each other (Additional file 6). Such phylogenetic incompatabilities may arise from either data ambiguity, convergent evolution or recombination events [62], suggesting that recombination may have played a strong role in shaping the cir repertoire.

\section{c) Identification of conserved amino acid motifs}

The identification of conserved amino acid sequences may provide indications of possible protein function(s). Multiple Expectation maximization for Motif Elicitation analysis (MEME, [37]) identified 14 motifs in the CIR amino acid sequences. Each motif is represented in Figure 2a as a WebLogo image [38], where the height of each letter indicates the proportion of CIR sequences containing that residue.

Motifs 1 and 3 were the most conserved, being present in $98.66 \%$ of CIR sequences. Motif 1 contained the almost unanimous YK residues, corresponding to the start of the third cir exon and part of the predicted CIR transmembrane domain. Motif 3 was found within the second cir exon and contained the highly conserved sequence YAILWLSY. Motif 10, present in $91.28 \%$ of CIRs, contained some degeneracy, but a clear conserved methionine indicated the $\mathrm{N}$-terminus of all CIR proteins. All CIRs possessing motif 10 also contained a cysteine six residues into the sequence. Conserved cysteine residues were also located within motifs 5, 7 and 14. Motif 10 was found at the N-terminus of almost all CIR proteins (91\%). Motifs 9, 3, 11, 5, 4 and 1 were also found in members of all sub-families.

The remaining motifs appeared to have sub-family specific distributions, with motifs 2,6 and 7 only found within sub-family B sequences. By contrast, motifs 8,12 , 13 and 14 were specific to members of CIR sub-family A. The arrangement of motifs within the amino acid sequence is indicated for a typical member of each major sub-family in Figure $2 b$.

Part of motif 8 (unique to sub-family A) lies within the predicted TM domain and the TM domains of Atype CIRs contain more proline residues than B-type CIRs. In addition the majority of CIRs containing more than one TM domain are found within sub-family A. Together, these differences indicate that the TM domain of CIR sub-type A proteins may have altered properties compared to the rest of the CIRs.

\section{d) Similarities between CIRs and RIFINS and function shift analysis}

The identification of two major CIR sub-families in Figure 1 resembled the $P$. falciparum RIFIN repertoire 


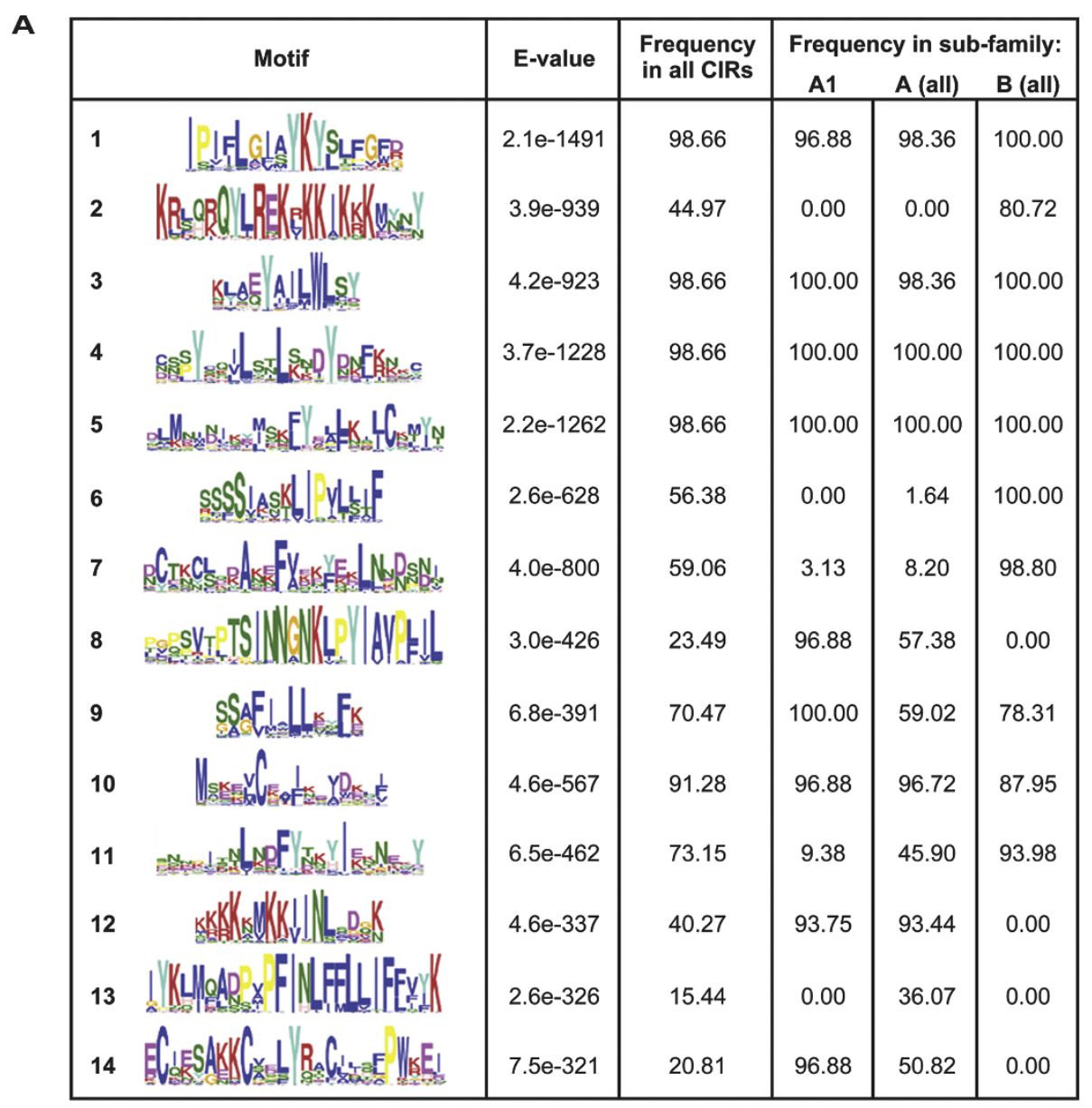

B

\begin{tabular}{|c|c|c|c|c|c|c|c|c|c|c|}
\hline A1-type CIR & 10 & 9 & 3 & & 5 & 14 & 4 & 8 & 1 & 12 \\
\hline B-type CIR & 10 & 9 & 3 & 11 & 5 & 7 & 4 & 6 & 1 & 2 \\
\hline
\end{tabular}

Figure 2 Conserved amino acid motifs within CIR sequences. Amino acid motifs present within the CIR repertoire were identified by MEME analysis (Bailey and Elkan, 1994). These are shown as Weblogo images [38], A). Hydrophobic residues are shown in blue, polar, non-charged residues in green, acidic residues in pink and positively charged residues in red. E-values refer to the significance of each motif found within the $\mathrm{CIR}$ sequences. The frequency (percentage) of CIR sequences within the whole repertoire, clade A1 or whole sub-families $\mathrm{A}$ and $\mathrm{B}$ containing each motif is shown. Examples of motif arrangement in CIR proteins are shown, B), using PCHAS_040110 and PCHAS_070130, which belong to the sub-families A (clade A1) and B, respectively. Exon: exon boundaries in the encoding cir genes are indicated by dotted lines.

organization [16,17]. As the rif and stevor multi-gene families have been suggested to be distantly related to the pir genes [18], we compared the CIR and RIFIN repertoires.

The RIFIN sub-family $A$ is defined by the presence of a 25 amino acid sequence, which is absent from RIFIN$B$ types [16,17]. An insertion sequence could be detected only in members of CIR sub-family A, which was most conserved in the clade A1. This was located between position 253 and 316 of the alignment of 183 CIRs (Additional file 7a). Furthermore, in this insertion (displayed as a weblogo, [38]), showed some similarities with the A-type RIFIN insertion sequence (Additional file $6 \mathrm{~b}$ and $\mathrm{c},[16,17])$. Approximately a third of the residues in each insertion sequence were hydrophobic and very few basic residues were present. Notably, both sequences included two conserved cysteine residues.

The two RIFIN sub-families have been shown to display different sub-cellular localizations [16], and thus Aand B-type RIFINs may have different functions. This hypothesis has been supported by bio-informatic analysis of the whole RIFIN repertoire in the P. falciparum 
clones 3D7, DD2 and HB3 [17]. To investigate whether the members of each major CIR sub-type could also have functionally diverged, the alignment of 183 CIRs was split into the two major sub-families, between which Rate- and Conservation- Shifting Sites were compared (RSS and CSS, respectively), as previously described $[17,49]$. Briefly, RSS measures the probability that each position in the alignment has a different mutation rate in the two sub-families, whilst CSS compares the amino acid distribution between members of each sub-family. Significant CSS and RSS sites are plotted in Figure 3a. 77 RSS (15.4\% of all positions) and 158 CSS (31.8\% of all positions) were identified along the alignment, strongly suggesting that functional divergence between the CIR sub-families may have occurred according to the criteria generated with protein families of known function [36].

The most significant CSS and RSS sites above the threshold indicated in Figure 3a are highlighted in a section of the CIR amino acid alignment, Figure 3b. Notably, several of the sites had altered conservation of cysteine residues (for example: at site 210 a conserved cysteine residue was present in sub-family A CIRs, but the residues $\mathrm{N}, \mathrm{K}, \mathrm{S}, \mathrm{G}$ or $\mathrm{D}$ could be found at this position in members of sub-family B, Table 1) or changes in residue conservation within the predicted TM domain, between residues 330-352 (at sites 330, 349 and 350, Figure $3 \mathrm{~b}$ and Table 1). Similar shifts have also been observed between the RIFIN A and B sub-families, with four highly significant shifts in cysteine conservation, and seven shifted sites detected within the RIFIN TM domain [17].

\section{II) Analysis of cir gene expression during plasmodium chabaudi infection a) RNA sequencing analysis}

To establish whether the different subgroups of CIR might encode for proteins with different biological functions, we determined the patterns of cir gene expression during an infection. For this, mRNA was purified from Plasmodium chabaudi AS iRBCs at the late trophozoite stage from four BALB/c mice and sequenced using Illumina/Solexa technology [Array Express accession number: E-ERAD-25 and ENA Study Accession Number: ERP000983]. Results obtained from intergenic regions allowed the determination of a threshold of detection, above which a gene was considered significantly expressed in each sample (Additional file 10). Accordingly, the expression of up to $40 \%$ of cir genes could be detected in each sample analyzed (Figure 4a).

In addition, mRNA was also sequenced from parasites passaged in two C57BL/6 mice. Both the BALB/c and C57BL/6 inbred strains of mice are commonly used hosts for $P$. chabaudi infection (for example: [68-71]), thus it was important to determine whether either host genetic background could influence cir expression. While in the C57BL/6 samples only $17 \%$ and $12 \%$ of the cir repertoire were expressed above background, these lower values were at least partly the result of a higher RPKM cutoff due to relatively high expression of

A
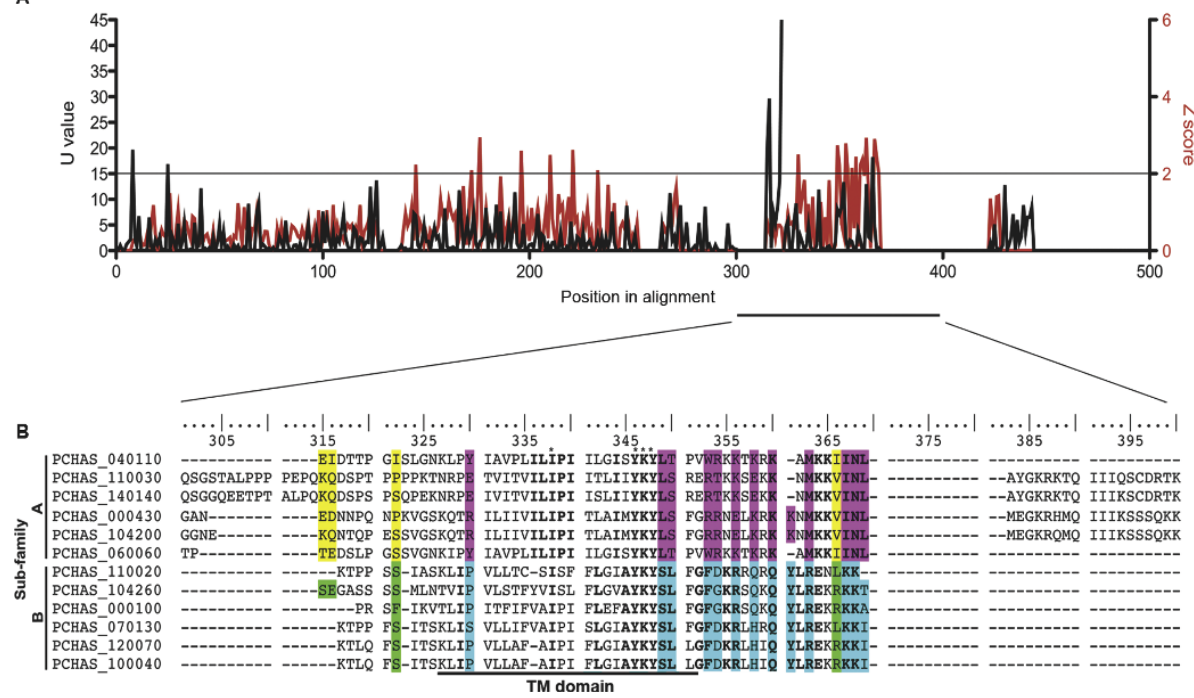

Figure 3 Funshift analysis of CIR sub-families A and B. Significant rate shifting sites (RSS, defined as $U$ values greater than 4) and conservation shifting sites (CSS, defined as Z scores greater than 0.5) are plotted along the CIR alignment, A). The sequences of 5 representative CIR sub-family A and B members are shown, B), with the most significantly shifting sites highlighted as follows: $U$ values greater than 15 are shown in yellow for sub-family $A$ and green for sub-family B members; Z scores greater than 2 are shown in pink for sub-family $A$ and blue for sub-family B members. The most significantly shifting sites, (as defined in B), are listed in Table 1. 
Table 1 Highly significant rate and conservation shifting sites identified between the two major CIR sub-families

\begin{tabular}{|c|c|c|c|}
\hline \multirow[t]{2}{*}{ Position in alignment } & \multicolumn{2}{|c|}{ Residues in CIR sub-family: } & \multirow[b]{3}{*}{ Z score } \\
\hline & A & B & \\
\hline \multicolumn{2}{|c|}{ Significant conservation shifting sites (CSS): } & & \\
\hline 145 & GWSRHYINLQD & K & 2.24 \\
\hline 172 & 1 & L & 2.09 \\
\hline 176 & IVLFS & Y & 2.95 \\
\hline 196 & C SWKLFYIQ & $F L \mid N$ & 2.60 \\
\hline 210 & $\mathrm{C}$ & N KS GD & 2.49 \\
\hline 221 & K & $S L F$ & 2.62 \\
\hline 233 & I A VTSMNYKHL & CFRYL & 2.09 \\
\hline 330 & YERGVKA I R & $P S A L T$ & 2.50 \\
\hline 349 & $\mathrm{~L}$ & S & 2.74 \\
\hline 350 & STKALVPYFI & L & 2.21 \\
\hline 353 & WR Q S I G C & F & 2.79 \\
\hline 354 & RTKGAVN & $\mathrm{DGH}$ & 2.09 \\
\hline 356 & KEMNTY & R & 2.15 \\
\hline 358 & KEFTNI & Q HKR N & 2.44 \\
\hline 360 & K & Q & 2.21 \\
\hline 361 & K & Y & 2.36 \\
\hline 363 & M & $\mathrm{R}$ & 2.93 \\
\hline 367 & 1 & K & 2.90 \\
\hline 368 & N & K & 2.42 \\
\hline 369 & $\mathrm{~L}$ & TA I KV & 2.01 \\
\hline \multicolumn{2}{|c|}{ Significant rate shifting sites (RSS): } & & $\mathrm{U}$ value \\
\hline 8 & M & M D KTNIL & 19.66 \\
\hline 25 & VNKGA & TYDVSEKNQPGI & 16.86 \\
\hline 315 & EKTASQPISDRLYGN & EPF LT & 19.99 \\
\hline 316 & I Q DENPASGHLKVTR & SEDGQR & 29.63 \\
\hline 322 & I PSVTNAKEQLCYHG & SFNTPL & 45.64 \\
\hline 366 & IVLD & LR V I P & 18.26 \\
\hline
\end{tabular}

The most significant CSS $(Z>2)$ and RSS $(U>15)$ are listed, with the possible amino acids for each of the two sub-families at each position of the alignment. $\times$ refers to the IUPAC code, representing any amino acid.

annotated introns and thus probably do not reflect differences in cir expression between BALB/c and C57BL/6 mice.

A list of the ten most highly expressed cir genes was established for each sample (Figure 4b). As their expression was most highly detected in a population of parasites (either due to higher levels of expression in selected iRBCs or expression in a higher number of iRBCs), these will henceforth be referred to as "dominant cirs". When we compared the lists of dominant cirs thus established, it was evident that most of these genes (> 60\%) were different between the samples analyzed (Figure 4c). This indicates that the relative level of cir transcripts may vary from one mouse to another; which could reflect antigenic variation, immune selection of iRBCs or sampling differences upon infection of the mice. Whilst the overall level of cir expression was significantly lower than for other parasite genes (Kolmogorov-Smirnov test; $\mathrm{D}=0.6742, \mathrm{P}$-value $<2.2 \mathrm{e}-16$,
Figure 4d), the dominant cir transcripts were found amongst the most highly expressed parasite genes.

Surprisingly, the same gene (PCHAS_110030) was expressed more than ten fold higher than any other cir genes in all the BALB/c samples analyzed. Similarly, three other cirs (PCHAS_140140, PCHAS_070130, and PCHAS_000430) were also dominant in these four samples. Furthermore, all of these genes, except PCHAS_140140, were dominant in at least one of the two C57BL/6 mice studied (Additional file 9). This indicates that some cir genes are consistently highly represented in a population of Plasmodium chabaudi AS parasites at the late trophozoite stage during the peak of parasitemia (8 days post infection).

To evaluate whether there was a relationship between CIR sub-families and functions, a comparison of their expression patterns was undertaken. In our analysis, more of the expressed cirs belonged to sub-family B (on average, $57.2 \% \pm 4.7 \%$ were B-type cirs and $42.3 \% \pm$ 
A

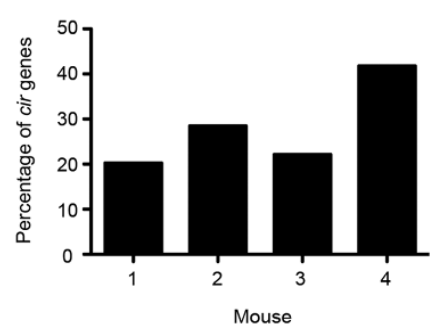

C

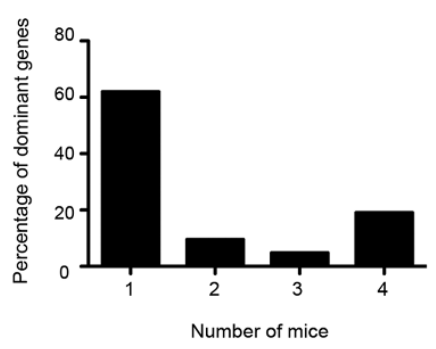

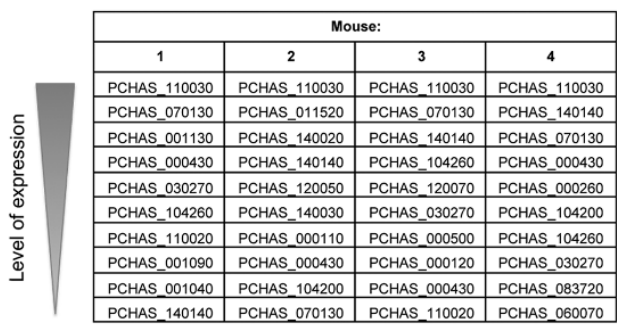

D

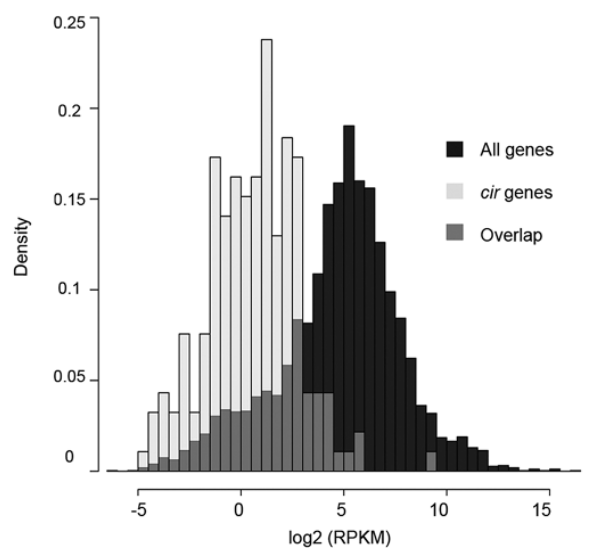

Figure 4 cir gene expression during infection. The percentage of cir genes for which transcripts were detected by RNA sequencing is shown for the four $P$. chabaudi infected BALB/C mice, A). The ten dominant cirs were tabulated from each infected mouse, ranked according to expression level, B). The number of $P$. chabaudi infected BALB/C mice for which a dominant cir transcript was detected is plotted, C). The densities of all $P$. chabaudi transcripts and cir transcripts are plotted against the $\log _{2}$ RPKM, a measure of transcript abundance, coloured in black and pale grey respectively, D). Regions of overlap are dark grey.

4.7\% were A-types; Figure 5a). A similar result was obtained in both analyzed C57BL/6 mice $(58.33 \% \pm$ $8.41 \%$ of cirs belonged to sub-family B and $41.66 \% \pm$ $8.41 \%$ were A-type cirs, data not shown). When the expression levels of cir genes from each sub-family were compared, no statistically significant differences could be detected ( $\mathrm{K}-\mathrm{S}$ test, $\mathrm{P}=0.46)$. It thus seems that the overall level of expression between cir genes belonging to sub-families A and B was similar.

However, the most highly expressed cirs were all members of sub-family A (Figure 5b). Indeed, PCHAS_110030, the most highly expressed cir gene in the six samples analyzed, belonged to this sub-family. We investigated whether this was also the case for the other dominant genes identified previously, Figure 4b. However, in each sample analyzed, about half of the dominant cir genes identified belonged to each subfamily (on average, $52.5 \% \pm 18.9 \%$ of the dominant genes were A-type cirs and $47.5 \% \pm 18.9 \%$ were members of sub-family B, Figure 5c, indicated by black dots in Additional file 3). This observation was confirmed in the C57BL/ 6 mice (on average, $50 \% \pm 14.14 \%$ of the dominant cirs belonged to sub-family A and 50\% \pm $14.14 \%$ were B-type cirs).

\section{b) Microarray analysis}

Timing of transcription could be an alternative mechanism by which CIRs of different subgroups mediate different functions, therefore we investigated the transcriptional pattern of these genes throughout the intra-erythrocytic developmental cycle (IDC) in P. chabaudi. For this purpose parasites were collected at 2hour intervals from mice with a synchronous infection of $P$. chabaudi for a total of $24 \mathrm{hrs}$, representing one complete IDC of the parasite. The RNA was extracted and cir transcription was determined using the panrodent Plasmodium spp microarray, which has been described previously [55].

Figure 6 shows the detected cir expression patterns throughout the IDC, where each time-point represents parasites collected from a single mouse [accession number in GEA: GSE33333]. Of the total 96 unique cir genes represented on the array, 49 showed clear transcriptional activation throughout the IDC. Each cir showed a single peak of transcription in line with previous reports that in Plasmodium, genes are only activated once during the IDC $[24,56]$. Importantly, not all cir genes were activated at the same time but rather showed progressive transcriptional activation throughout 


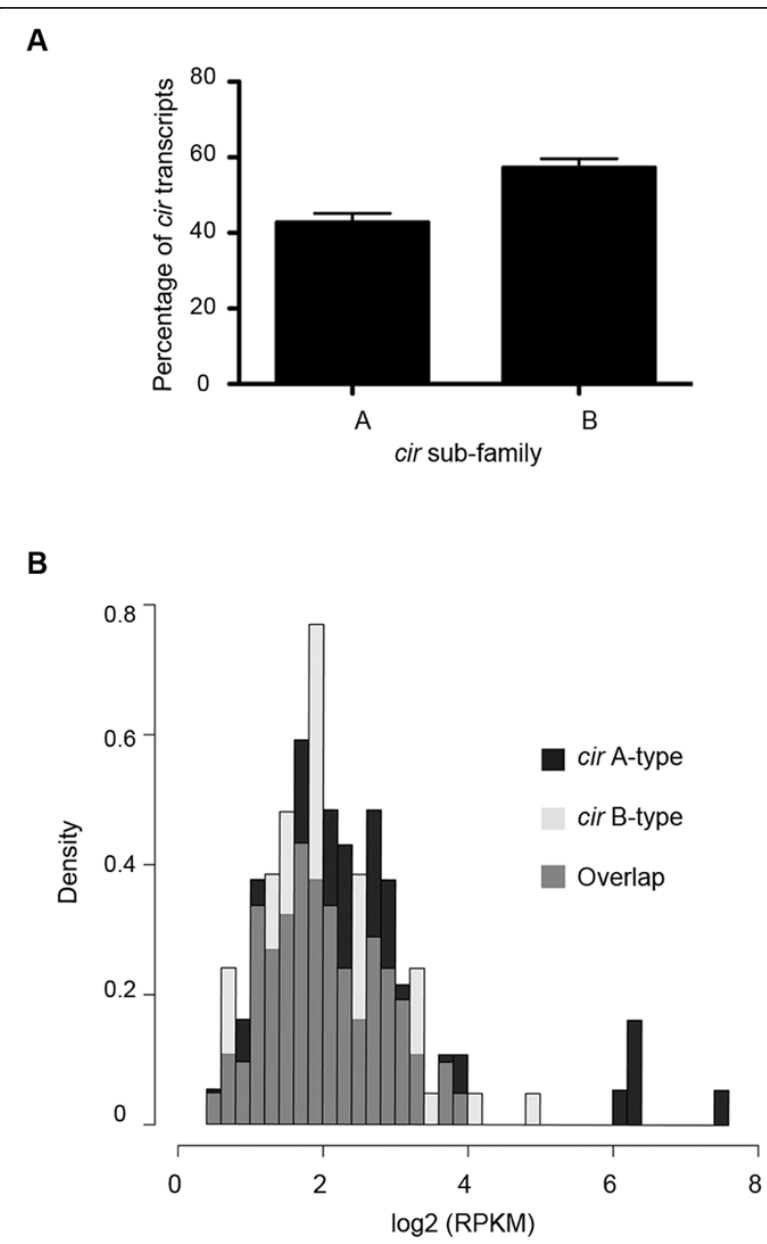

C

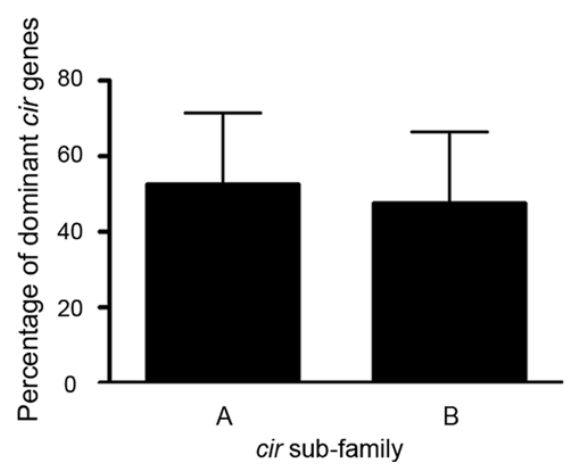

Figure 5 Comparison of A- and B-type cir expression during $P$. chabaudi infection. The percentage of cir transcripts belonging to sub-families A and B is shown, A). The mean of the four BALB/C mice is plotted; error bars represent the standard error of the mean. The density of cir transcripts is plotted against the $\log _{2}$ RPKM, B). Transcripts belonging to cir sub-families $A$ and $B$ are coloured in black and pale grey, respectively. Regions of overlap are coloured dark grey. The percentage of dominant cir transcripts belonging to sub-families A and B is shown, C). The mean of the four BALB/C mice is plotted; error bars represent the standard error of the mean.
A
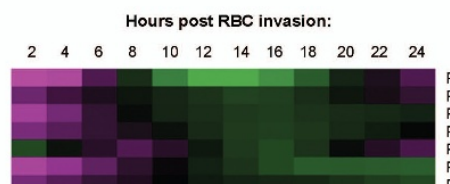
cir gene
PCHAS 140040 BA
PCHAS_ 140040 PCHAS_001010 PCHAS 010040 PCHAS 090030 PCHAS 000120 PCHAS 0010030 PCHAS 070060 PCHAS 030160 A1 PCHAS 120040 PCHAS 041960 PCHAS 030190 PCHAS 140130 PCHAS_146870 Á PCHAS 146840 PCHAS 011450 PCHAS 000950 PCHAS_010120 PCHAS 060110 PCHAS 000730 PCHAS 090010 PCHAS 001130 PCHAS 060120 PCHAS 000390 PCHAS 110030 PCHAS 114730 PCHAS 050020 PCHAS 001090 PCHAS_014990 PCHAS 110020 PCHAS 000110 PCHAS 130110 CHAS 140090 PCHAS 070130 PCHAS_01150 PCHAS 13017 PCHAS 13008 PCHAS_130100

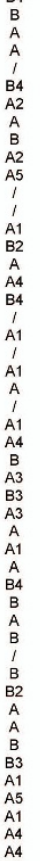

B

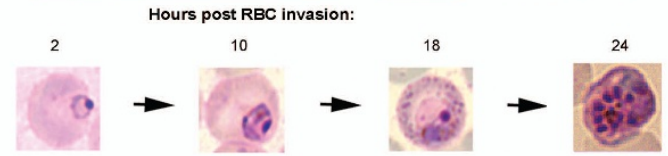

Figure 6 Transcriptional profile of $\mathbf{4 9}$ detected cir genes throughout the IDC. 49 cir transcripts could be detected by microarray, which are listed along with sub-family classifications, next to their pattern of expression throughout the IDC, A). Black shading indicates no change in cir expression for the IRBC population, whilst magenta and green shading indicate higher and lower levels of cir transcripts, respectively. Unclassified cir genes are denoted by/. Representative parasite stages from four of the timepoints throughout the $24 \mathrm{hr}$ IDC are shown, B).

the IDC with approximately one third of the analyzed cirs respectively showing peak transcriptional activity at the ring, trophozoite and schizont stages. Similar observations have also been made in $P$. vivax, where members of the vir gene family showed a comparable transcription pattern [24].

Differences in absolute levels of transcription of the two cir sub-families could not be quantified using this approach as two-channel hybridization only allows relative abundance comparison for each gene and not between different genes. However, many members of each sub-family could be detected at every time point. These data also strongly suggest that different cirs are indeed transcribed at different stages of parasite development in the erythrocyte in line with their products having different functions. 
Interestingly, several of the dominant cir transcripts from the RNA sequencing experiments were also detected in this microarray timecourse, despite the fact that this material was obtained from separate $P$. chabaudi infections. These cirs were: PCHAS_110030, PCHAS_140140, PCHAS_110020, PCHAS_001090, PCHAS_070130, PCHAS_000110 and PCHAS_000120, which comprised approximately a third of the dominant cirs identified by RNA sequencing analysis. In addition, as each time point represents the material from a single mouse, the progression of cir transcription appeared to be conserved in the mice that were infected with a single starting parasite population and would suggest that limited switching occurs within a single cycle of asexual development. Importantly, the peak timing of expression of these cirs detected by microarray is consistent with the parasite stage used for the RNA sequencing analysis, further supporting the notion that the timing of expression of different CIR is conserved in different independent infections.

\section{Discussion}

In total, $196 \mathrm{cir}$ genes have been identified and annotated in the $P$. chabaudi genome. The cir genes distribute into two major groups, according to sequence similarity. Many cirs from both sub-families are transcribed during $P$. chabaudi infection, although dominant cir transcripts are present. Differences between the subfamilies, such as sub-family specific amino acid motifs and altered residue conservation, particularly within the predicted TM domain, indicate that the two sub-families could be functionally divergent. Recombination of cir genes may have occurred within the cir repertoire, consistent with that observed in other Plasmodium multigene families including var and sicavar (for example: $[63,64])$.

Similar to the VIR and YIR gene families [2,20,22,23], we confirm that there are also sub-groups within the CIR repertoire in agreement with Ebbinghaus and Krucken [30]. As each of the major groups defined here represented almost half of the CIRs, we defined these as the sub-families: A, comprising the more divergent CIRs; and B, containing more conserved CIRs. These CIR sub-families could each be further sub-divided into smaller clades. Despite the different analysis of CIRs recently described [30], the same groups could be identified within our network (as clade A1 and sub-family B), indicating that the sequences in each of these groups are indeed highly similar to each other. While the overall conservation of PIRs across species is relatively low, some evidence exists for sub-families being shared between $P$. yoelii and P. berghei [23], as well as P. chabaudi, P. yoelii and P. berghei [30] suggesting common functions.
The extensive sequence variation often seen in multigene families like var, rif and stevor in $P$. falciparum, or the pirs in $P$. vivax and rodent malaria parasites, is thought to reflect that these genes are under immune pressure (eg. [2,29,72-74]). In our analysis however, we have identified 14 conserved amino acid motifs, suggesting that some parts of the CIR sequences are under constraints important for the biological functions of these proteins. While some motifs were found in the majority of CIRs, motifs unique to each of the two sub-families were also detected. This divergence of conserved amino acid sequence motifs in the different sub-groups presents strong evidence that the different CIR sub-types may indeed carry out distinct but related functions in the parasite, as suggested also for PIR and RIFIN sequences [18]. Since the CIR A sub-family contained the most divergent CIRs, these proteins may be exposed to stronger selective pressure, and it is possible that their diverse nature enables immune evasion in vivo.

In line with the functional diversification of CIR subtypes $\mathrm{A}$ and $\mathrm{B}$, some CIRs belonging to sub-type A possessed a unique amino acid motif (motif 8 ) at the beginning of the predicted TM domain. This, in addition to different conservation of proline residues and other amino acids within the predicted TM domains of each sub-family, suggests that CIR sub-family A proteins may have a functionally different TM domain than the B-type CIRs, perhaps altering which membrane the proteins are able to span.

Janssen and colleagues have predicted that rif and stevor could be ancestral to the pir genes due to similarities in terms of size, amino acid motifs and conservation of the first intron [18]. The observations that the CIR repertoire could be separated into two major sub-types namely thanks to an insertion within CIR sub-type A, a section of which contained two highly conserved cysteine residues [similar to RIFIN sub-type A, [16,17]], is strong evidence that the CIR family displays further parallels to the RIFINs. The variation we observed in the number and position of cysteine residues in the two CIR sub-families could significantly impact on the overall protein tertiary structure, and thereby function. As predicted for the A and B type RIFINs $[17,49]$, we found high proportions of rate and conservation shifting sites between the two major CIR sub-families, providing further support for the idea of functional divergence.

Transcriptional differences in both in amount as well as timing can indicate functional differences between related genes. Until now, only Northern blot, degenerate PCR and restriction fragment length polymorphism (RFLP) analyses have been used to give indications of cir gene expression during $P$. chabaudi infection $[29,30]$. Here, we have used both mRNA sequencing as well as microarray studies to obtain a detailed picture of cir 
transcription in vivo. The expression of up to $40 \%$ of the cir genes could be detected during an infection. Different cirs were transcribed at different times during the IDC of the parasite, with distinct cirs being transcribed in ring, trophozoite and schizont stages. The timing of transcription for a specific cir appeared to be conserved from one animal to another (as seen in the microarray experiments) as well as across different experiments (as seen with the good overlap between the genes transcribed in the trophozoite stage as detected by both RNA sequencing and microarray), suggesting that CIRs may carry out distinct ring-, trophozoite-or schizontspecific functions. This is further supported by the observation that pir transcription follows a similar pattern in both $P$. vivax and P. yoelii, where $59 \%$ of the virs and $42 \%$ of the yirs were expressed respectively $[4,24]$.

Whilst most cir genes were transcribed at lower levels than other parasite genes, this most likely reflects differences in the proportion of parasites that actually express a particular cir, resulting in populations of iRBCs each expressing different $c i r$ genes. This would be consistent with observations made for virs, yirs and vars $[1,4,75]$, that individual iRBCs transcribe only one or a small number of these genes. It is therefore of particular interest that three cir genes were represented at relatively high levels in all six samples analyzed by RNA sequencing (PCHAS_110030, PCHAS_070130, and PCHAS_000430), with PCHAS_110030 being detected at a level more than 10 fold higher than any other cir in all the samples analyzed. In a classic model of antigenic variation, it would be expected that a single or relatively small number of a gene family would be transcribed in any population, with other members of the gene family being transcribed at much lower levels. This is exactly what we have detected during P. chabaudi infection.

While our data suggest that cirs belonging to subfamily A are more highly transcribed than those belonging to sub-family B, these differences are relatively small. At this stage there it is not clear whether there is a preference for the transcription of A or B sub-families. What is evident though, is that both A and B sub-family members were transcribed at the peak of a blood-stage $P$. chabaudi infection. Considering that the sequence analysis carried out here strongly suggests functional differences between the two sub-types, the expression of both sub-families may indicate that A and B-type CIRs provide complementary but non-overlapping roles at different stages during infection.

\section{Conclusions}

We have here presented a thorough analysis of the cir repertoire, highlighting similarities between these genes and other multi-gene families. The two CIR sub-families have been predicted to have different functions, as shown for the RIFINs of P. falciparum. Whilst these function(s) remain unknown, the large number of cir genes and their expression throughout the IDC indicates that CIR proteins are likely to play key roles in the biology of the parasite. These may include antigenic variation and immune evasion. Our application of RNA sequencing during $P$. chabaudi infection has enabled the detection of dominant cir transcripts for the first time, supporting such roles. Further application of these methodologies may elucidate the functions of CIR proteins and help to clarify the roles that members of the CIR sub-families play during infection.

\section{Additional material}

Additional file 1: Alignment of 183 CIR sequences.

Additional file 2: CIR alignment conservation.

Additional file 3: CIR network showing bootstrap values.

Additional file 4: Maximum likelihood tree of CIR sequences.

Additional file 5: Sub-families identified from the alignment of 183 CIRs.

Additional file 6: Detection of phylogenetic incompatibilities between cir genes.

Additional file 7: Identification of similarities between the CIR and RIFIN repertoires.

Additional file 8: Raw microarray data.

Additional file 9: Raw RNA sequencing data.

Additional file 10: cir gene expression threshold of detection determination.

Additional file 11: Legends to Additional Files 1-10

\section{Acknowledgements}

We are grateful to the PFAM group at the Wellcome Trust Sanger Institute for creating a HMM based on the cir genes, and to Saraswathi Abhiman and Erik L. Sonnhammer for allowing us to use the Funshift program in our analysis [49].

Funding

This work was supported by the Medical Research Council, UK (file reference U117584248); Singapore A*Star-UK MRC Joint Grant (A*Star reference 10/1/ 22/24/630); Singapore Nanyang Technological University (WBS $N^{\circ}$ M47080081); and the European Community's Seventh Framework Programme (FP7/2007-2013) under grant agreement $N^{\circ}$ 242095. Jennifer Lawton was in receipt of an MRC PhD scholarship.

\section{Author details}

${ }^{1}$ Division of Parasitology, MRC National Institute for Medical Research, London, UK. ${ }^{2}$ Division of Genomics and Genetics, Nanyang Technological University Singapore, Singapore. ${ }^{3}$ Parasite Genomics, Wellcome Trust Sanger Institute, Hinxton, UK. ${ }^{4}$ Pathogen Genomics Group, Computational Bioscience Research Center, Chemical Life Sciences and Engineering Division, 4700 King Abdullah University of Science and Technology, Thuwal 23955-6900, Kingdom of Saudi Arabia.

\section{Authors' contributions}

$J^{(1 \text { st author) }}$ carried out the cir gene annotation in collaboration with UB, AP and $M B$; carried out the bioinformatic analyses, supervised by AJ; prepared RNA samples for RNAseq and drafted the manuscript in collaboration with TB. RNAseq data were analyzed by AJR, TDO and TB. Microarray analyses were performed by YXY and PP. JL ${ }^{\text {(last author) }}$ conceived of and supervised 
the study, and edited the manuscript with DC, AJ, AJR, AP, MB and PP. All authors read and approved the final manuscript.

\section{Competing interests}

The authors declare that they have no competing interests.

Received: 4 November 2011 Accepted: 29 March 2012

Published: 29 March 2012

\section{References}

1. Joergensen $L$, Bengtsson DC, Bengtsson A, Ronander E, Berger SS, Turner L, Dalgaard MB, Cham GK, Victor ME, Lavstsen T, et al: Surface co-expression of two different PfEMP1 antigens on single plasmodium falciparuminfected erythrocytes facilitates binding to ICAM1 and PECAM1. PLOS Pathogens 2010, 6(9):e1001083.

2. del Portillo HA, Fernandez-Becerra C, Bowman S, Oliver K, Preuss M, Sanchez CP, Schneider NK, Villalobos JM, Rajandream MA, Harris D, et al: A superfamily of variant genes encoded in the subtelomeric region of Plasmodium vivax. Nature 2001, 410(6830):839-842.

3. Howard RJ, Barnwell JW, Kao V: Antigenic variation of Plasmodium knowlesi malaria: identification of the variant antigen on infected erythrocytes. Proc Natl Acad Sci USA 1983, 80(13):4129-4133.

4. Cunningham D, Fonager J, Jarra W, Carret C, Preiser P, Langhorne J: Rapid changes in transcription profiles of the Plasmodium yoelii yir multigene family in clonal populations: lack of epigenetic memory? PLoS One 2009, 4(1):e4285.

5. Gilks CF, Walliker D, Newbold Cl: Relationships between sequestration, antigenic variation and chronic parasitism in Plasmodium chabaudi chabaudi-a rodent malaria model. Parasite Immunology 1990, 12(1):45-64

6. Rowe JA, Claessens A, Corrigan RA, Arman M: Adhesion of Plasmodium falciparum-infected erythrocytes to human cells: molecular mechanisms and therapeutic implications. Expert Reviews in Molecular Medicine 2009, 11:e16.

7. Scherf A, Lopez-Rubio JJ, Riviere L: Antigenic variation in Plasmodium falciparum. Annu Rev Microbiol 2008, 62:445-470.

8. Marsh K, Howard RJ: Antigens induced on erythrocytes by $P$. falciparum: Expression of diverse and conserved determinants. Science 1986 , 231(4734):150-153

9. Marsh K, Otoo L, Hayes RJ, Carson DC, Greenwood BM: Antibodies to blood stage antigens of Plasmodium falciparum in rural Gambians and their relation to protection against infection. Transactions of the Royal Society of Tropical Medicine and Hygiene 1989, 83(3):293-303.

10. Fischer K, Chavchich M, Huestis R, Wilson DW, Kemp DJ, Saul A: Ten families of variant genes encoded in subtelomeric regions of multiple chromosomes of Plasmodium chabaudi, a malaria species that undergoes antigenic variation in the laboratory mouse. Mol Microbiol 2003, 48(5):1209-1223.

11. Rubio JP, Thompson JK, Cowman AF: The var genes of Plasmodium falciparum are located in the subtelomeric region of most chromosomes. EMBO J 1996, 15(15):4069-4077.

12. Cunningham D, Lawton J, Jarra W, Preiser $P$, Langhorne J: The pir multigene family of Plasmodium: antigenic variation and beyond. $\mathrm{Mo}$ Biochem Parasitol 2010, 170(2):65-73.

13. Bachmann A, Predehl S, May J, Harder S, Burchard GD, Gilberger TW, Tannich E, Bruchhaus I: Highly co-ordinated var gene expression and switching in clinical Plasmodium falciparum isolates from non-immune malaria patients. Cellular Microbiology 2011, 13(9):1397-409.

14. Kraemer SM, Smith JD: Evidence for the importance of genetic structuring to the structural and functional specialization of the Plasmodium falciparum var gene family. Mol Microbiol 2003 50(5):1527-1538

15. Lavstsen T, Salanti A, Jensen AT, Arnot DE, Theander TG: Sub-grouping of Plasmodium falciparum 3D7 var genes based on sequence analysis of coding and non-coding regions. Malar J 2003, 2:27.

16. Petter $M$, Haeggstrom $M$, Khattab A, Fernandez V, Klinkert MQ, Wahlgren M: Variant proteins of the Plasmodium falciparum RIFIN family show distinct subcellular localization and developmental expression patterns. $\mathrm{Mol}$ Biochem Parasitol 2007, 156(1):51-61.

17. Joannin N, Abhiman S, Sonnhammer EL, Wahlgren M: Sub-grouping and sub-functionalization of the RIFIN multi-copy protein family. BMC Genomics 2008, 9:19.
18. Janssen CS, Phillips RS, Turner CM, Barrett MP: Plasmodium interspersed repeats: the major multigene superfamily of malaria parasites. Nucleic Acids Res 2004, 32(19):5712-5720.

19. Pain A, Bohme $U$, Berry AE, Mungall K, Finn RD, Jackson AP, Mourier $T$, Mistry J, Pasini EM, Aslett MA, et al: The genome of the simian and human malaria parasite Plasmodium knowlesi. Nature 2008, 455(7214):799-803.

20. Carlton JM, Adams JH, Silva JC, Bidwell SL, Lorenzi H, Caler E, Crabtree J, Angiuoli SV, Merino EF, Amedeo P, et al: Comparative genomics of the neglected human malaria parasite Plasmodium vivax. Nature 2008 455(7214):757-763.

21. del Portillo HA, Lanzer M, Rodriguez-Malaga S, Zavala F, FernandezBecerra C: Variant genes and the spleen in Plasmodium vivax malaria. Int J Parasitol 2004, 34(13-14):1547-1554.

22. Merino EF, Fernandez-Becerra C, Durham AM, Ferreira JE, Tumilasci VF, d'Arc-Neves J, da Silva-Nunes M, Ferreira MU, Wickramarachchi T, UdagamaRandeniya $P$, et al: Multi-character population study of the vir subtelomeric multigene superfamily of Plasmodium vivax, a major human malaria parasite. Mol Biochem Parasitol 2006, 149(1):10-16.

23. Fonager J, Cunningham D, Jarra W, Koernig S, Henneman AA, Langhorne J, Preiser $P$ : Transcription and alternative splicing in the yir multigene family of the malaria parasite Plasmodium y. yoelii: identification of motifs suggesting epigenetic and post-transcriptional control of RNA expression. Molecular and Biochemical Parasitology 2007, 156(1):1-11.

24. Bozdech Z, Mok S, Hu G, Imwong M, Jaidee A, Russell B, Ginsburg H, Nosten F, Day NP, White NJ, et al: The transcriptome of Plasmodium vivax reveals divergence and diversity of transcriptional regulation in malaria parasites. Proc Natl Acad Sci USA 2008, 105(42):16290-16295.

25. McLean SA, Pearson CD, Phillips RS: Plasmodium chabaudi: antigenic variation during recrudescent parasitaemias in mice. Exp Parasitol 1982, 54(3):296-302.

26. McLean SA, Pearson CD, Phillips RS: Plasmodium chabaudi: relationship between the occurrence of recrudescent parasitaemias in mice and the effective levels of acquired immunity. Exp Parasitol 1982, 54(2):213-221.

27. McLean SA, Pearson CD, Phillips RS: Antigenic variation in Plasmodium chabaudi: analysis of parent and variant populations by cloning. Parasite Immunology 1986, 8(5):415-424.

28. Mota MM, Jarra W, Hirst E, Patnaik PK, Holder AA: Plasmodium chabaudiinfected erythrocytes adhere to CD36 and bind to microvascular endothelial cells in an organ-specific way. Infect Immun 2000, 68(7):4135-4144

29. Janssen CS, Barrett MP, Turner CM, Phillips RS: A large gene family for putative variant antigens shared by human and rodent malaria parasites. Proceedings of the Royal Society B: Biological Sciences 2002, 269(1489):431-436

30. Ebbinghaus $P$, Krucken J: Characterization and tissue-specific expression patterns of the Plasmodium chabaudi cir multigene family. Malaria Journal 2011, 10:272

31. Rutherford K, Parkhill J, Crook J, Horsnell T, Rice P, Rajandream MA, Barrell B: Artemis: sequence visualization and annotation. Bioinformatics 2000 16(10):944-945.

32. Carver T, Berriman M, Tivey A, Patel C, Bohme U, Barrell BG, Parkhill J, Rajandream MA: Artemis and ACT: viewing, annotating and comparing sequences stored in a relational database. Bioinformatics 2008, 24(23):2672-2676.

33. The Plasmodium chabaudi homepage in GeneDB. [http://www.genedb. org/Homepage/Pchabaudi]

34. Hall N, Karras M, Raine JD, Carlton JM, Kooij TWA, Berriman M, Florens L, Janssen CS, Pain A, Christophides GK, et al: A comprehensive survey of the Plasmodium life cycle by genomic, transcriptomic, and proteomic analyses. Science 2005, 307(5706):82-86.

35. Finn RD, Mistry J, Tate J, Coggill P, Heger A, Pollington JE, Gavin OL, Gunasekaran P, Ceric G, Forslund K, et al: The Pfam protein families database. Nucleic Acids Res 2010, , 38 Database: D211-D222.

36. Sonnhammer EL, Eddy SR, Birney E, Bateman A, Durbin R: Pfam: multiple sequence alignments and HMM-profiles of protein domains. Nucleic Acids Res 1998, 26(1):320-322.

37. Bailey $\mathrm{TL}$, Elkan C: Fitting a mixture model by expectation maximization to discover motifs in biopolymers. Proceedings of the Second International Conference on Intelligent Systems for Molecular Biology 1994, 2:28-36. 
38. Crooks GE, Hon G, Chandonia JM, Brenner SE: WebLogo: a sequence logo generator. Genome Res 2004, 14(6):1188-1190.

39. Edgar RC: MUSCLE: multiple sequence alignment with high accuracy and high throughput. Nucleic Acids Res 2004, 32(5):1792-1797.

40. Huson DH: SplitsTree: analyzing and visualizing evolutionary data. Bioinformatics 1998, 14(1):68-73.

41. Bryant D, Moulton V: Neighbor-net: an agglomerative method for the construction of phylogenetic networks. Mol Biol Evol 2004, 21(2):255-265.

42. Gambette P, Huson DH: Improved layout of phylogenetic networks. IEEE/ ACM Transactions on Computational Biology and Bioinformatics 2008, 5(3):472-479.

43. Guindon S, Lethiec F, Duroux P, Gascuel O: PHYML Online-a web server for fast maximum likelihood-based phylogenetic inference. Nucleic Acids Res 2005, , 33 Web Server: W557-W559.

44. Le SQ, Gascuel O: An improved general amino acid replacement matrix. Mol Biol Evol 2008, 25(7):1307-1320.

45. Anisimova M, Gascuel O: Approximate likelihood-ratio test for branches: A fast, accurate, and powerful alternative. Syst Biol 2006, 55(4):539-552.

46. Carlton JM, Angiuoli SV, Suh BB, Kooij TW, Pertea M, Silva JC, Ermolaeva MD, Allen JE, Selengut JD, Koo HL, et al: Genome sequence and comparative analysis of the model rodent malaria parasite Plasmodium yoelii yoelii. Nature 2002, 419(3):512-519.

47. Milne I, Wright F, Rowe G, Marshall DF, Husmeier D, McGuire G: TOPALi: software for automatic identification of recombinant sequences within DNA multiple alignments. Bioinformatics 2004, 20(11):1806-1807.

48. Milne I, Lindner D, Bayer M, Husmeier D, McGuire G, Marshall DF, Wright F: TOPALi v2: a rich graphical interface for evolutionary analyses of multiple alignments on HPC clusters and multi-core desktops. Bioinformatics 2009, 25(1):126-127.

49. Abhiman S, Sonnhammer EL: FunShift: a database of function shift analysis on protein subfamilies. Nucleic Acids Res 2005, , 33 Database: D197-D200.

50. Knudsen B, Miyamoto MM: A likelihood ratio test for evolutionary rate shifts and functional divergence among proteins. Proc Natl Acad Sci USA 2001, 98(25):14512-14517.

51. Slade SJ, Langhorne J: Production of interferon-gamma during infection of mice with Plasmodium chabaudi chabaudi. Immunobiology 1989, 179(45):353-365.

52. Shinkaia Y, Rathbuna G, Lama KP, Oltza EM, Stewarta V, Mendelsohna M, Charrona J, Dattaa M, Younga F, Stalla AM, et al: RAG-2-deficient mice lack mature lymphocytes owing to inability to initiate $V(D) J$ rearrangement. Cell 1992, 68(5):855-867.

53. von der Weid T, Honarvar N, Langhorne J: Gene-targeted mice lacking B cells are unable to eliminate a blood stage malaria infection. J Immunol 1996, 156(7):2510-2516.

54. Kyes S: Reliable RNA preparation for Plasmodium falciparum. In Methods in Malaria Research.. Fourth edition. Edited by: Ljungström I, Perlmann $\mathrm{H}_{4}$ Schlichtherle M, Artur Scherf A, Wahlgren M. Manassas, Virginia; 2004:175-178.

55. Liew KJL, Hu G, Bozdech Z, Peter PR: Defining species specific genome differences in malaria parasites. BMC Genomics 2010, 11(1):128.

56. Bozdech Z, Llinas M, Pulliam BL, Wong ED, Zhu J, DeRisi JL: The transcriptome of the intraerythrocytic developmental cycle of Plasmodium falciparum. PLoS Biology 2003, 1(1):E5

57. Otto TD, Wilinski D, Assefa S, Keane TM, Sarry LR, Bohme U, Lemieux J, Barrell B, Pain A, Berriman M, et al: New insights into the blood-stage transcriptome of Plasmodium falciparum using RNA-Seq. Mol Microbiol 2010, 76(1):12-24.

58. Trapnell C, Pachter L, Salzberg SL: TopHat: Discovering splice junctions with RNA-Seq. Bioinformatics 2009, 25(9):1105-1111.

59. Mortazavi A, Williams BA, McCue K, Schaeffer L, Wold B: Mapping and quantifying mammalian transcriptomes by RNA-Seq. Nature Methods 2008, 5:621-628

60. Kolmogorov A: Sulla determinazione empirica di una legge di distribuzione. Giornale dell'Istituto Italiano degli Attuari 1933, 4:83-91.

61. Plotcon. [http://emboss.bioinformatics.nl/cgibin/emboss/plotcon].

62. Morrison DA: Networks in phylogenetic analysis: new tools for population biology. Int J Parasitol 2005, 35(5):567-582.

63. Corredor V, Meyer EV, Lapp S, Corredor-Medina C, Huber CS, Evans AG, Barnwell JW, Galinski MR: A SICAvar switching event in Plasmodium knowlesi is associated with the DNA rearrangement of conserved 3' noncoding sequences. Mol Biochem Parasitol 2004, 138(1):37-49.

64. Frank M, Kirkman L, Costantini D, Sanyal S, Lavazec C, Templeton TJ, Deitsch KW: Frequent recombination events generate diversity within the multi-copy variant antigen gene families of Plasmodium falciparum. Int J Parasitol 2008, 38(10):1099-1109.

65. Morell V: TreeBASE: the roots of phylogeny. Science 1996, 273:569-600

66. Piel W, Donoghue MJ, Sanderson MJ: TreeBASE: a database of phylogenetic knowledge. In To the interoperable "Catalog of Life" with partners Species 2000 Asia Oceanea Research Report from the National Institute for Environmental Studies No 171. Edited by: Shimura J, Wilson KL, Gordon D. Tsukuba, Japan; 2002:41-47.

67. Sanderson MJ, Donoghue MJ, Piel W, Eriksson T: TreeBASE: a prototype database of phylogenetic analyses and an interactive tool for browsing the phylogeny of life. American Journal of Botany 1994, 81(6):183.

68. Belyaev NN, Brown DE, Diaz AIG, Rae A, Jarra W, Thompson J, Langhorne J, Potocnik AJ: Induction of an IL7-R + c-Kit hi myelolymphoid progenitor critically dependent on IFN- $\gamma$ signaling during acute malaria. Nat Immunol 2010, 11(6):477-485.

69. Spence PJ, Cunningham D, Jarra W, Lawton J, Langhorne J, Thompson J: Transformation of the rodent malaria parasite Plasmodium chabaudi. Nat Protoc 2011, 6(4):553-561.

70. Sponaas AM, Do Rosario APF, Voisine C, Mastelic B, Thompson J, Koernig S, Jarra W, Renia L, Mauduit M, Potocnik AJ, et al: Migrating monocytes recruited to the spleen play an important role in control of blood stage malaria. Blood 2009, 114(27):5522-5531.

71. Stephens R, Langhorne J: Effector memory Th1 CD4 T cells are maintained in a mouse model of chronic malaria. PLoS Pathogens 2010, 6(11):e1001208.

72. Su XZ, Heatwole VM, Wertheimer SP, Guinet F, Herrfeldt JA, Peterson DS, Ravetch JA, Wellems TE: The large diverse gene family var encodes proteins involved in cytoadherence and antigenic variation of Plasmodium falciparum-infected erythrocytes. Cell 1995, 82(1):89-100

73. Kyes S, Horrocks P, Newbold C: Antigenic variation at the infected red cell surface in malaria. Annual Review of Microbiology 2001, 55:673-707.

74. Niang M, Xue YY, Preiser PR: The Plasmodium falciparum STEVOR multigene family mediates antigenic variation of the infected erythrocyte. PLoS Pathogens 2009, 5(2):e1000307.

75. Fernandez-Becerra C, Pein O, de Oliveira TR, Yamamoto MM, Cassola AC, Rocha C, Soares IS, de Braganca Pereira CA, del Portillo HA: Variant proteins of Plasmodium vivax are not clonally expressed in natural infections. Mol Microbiol 2005, 58(3):648-658.

doi:10.1186/1471-2164-13-125

Cite this article as: Lawton et al: Characterization and gene expression analysis of the cir multi-gene family of plasmodium chabaudi chabaudi (AS). BMC Genomics 2012 13:125.

\section{Submit your next manuscript to BioMed Central and take full advantage of:}

- Convenient online submission

- Thorough peer review

- No space constraints or color figure charges

- Immediate publication on acceptance

- Inclusion in PubMed, CAS, Scopus and Google Scholar

- Research which is freely available for redistribution

Submit your manuscript at www.biomedcentral.com/submit
C Biomed Central 